\title{
microRNAs become macro players in somatic cell reprogramming
}

Tamer T Onder, ${ }^{1,2}$ and George Q Daley*1,2

\begin{abstract}
Embryonic stem cell specific microRNAs (miRNAs) have previously been shown to enhance the efficiency of transcription-factor-based reprogramming. However, whether reprogramming could be achieved entirely by miRNAs remained unclear. A recent report shows that the expression of the miR-302/367 cluster of miRNAs can directly reprogram somatic cells without the use of any transcription factors. This new method raises interesting questions about the mechanisms of reprogramming and is likely to facilitate the generation of induced pluripotent stem cells for potential future clinical use.
\end{abstract}

\section{Transcription factor-based somatic cell reprogramming}

In 2006, Takahashi and Yamanaka [1] demonstrated that differentiated cells can be converted into induced pluripotent stem cells (iPSCs) by the expression of four transcription factors - Oct4, Sox2, Klf4 and c-Myc - which have been termed Yamanaka factors. From the perspective of basic cell biology, somatic cell reprogramming has radically altered our thinking on the plasticity of cell states. In addition, the derivation of iPSCs from numerous normal and diseased cell sources has enabled the generation of patient-specific stem cells for eventual use in cell therapy and regenerative medicine. A number of alternatives and refinements to the original four-factor reprogramming method have been devised over the years. These have included ectopic expression of alternative reprogramming factors, such as Nanog and Lin28,

\footnotetext{
*Correspondence: George.daley@childrens.harvard.edu 'Stem Cell Transplantation Program, Division of Pediatric Hematology/Oncology, Manton Center for Orphan Disease Research, Howard Hughes Medical Institute, Children's Hospital Boston and Dana Farber Cancer Institute, Division of Hematology, Brigham and Women's Hospital, Boston, MA 02115, USA 2Department of Biological Chemistry and Molecular Pharmacology, Harvard Medical School, Harvard Stem Cell Institute, Boston, MA 02115, USA
}

manipulation of pathways that act as barriers to reprogramming, such as p53 and p21, transient expression of reprogramming proteins to avoid stable genetic modification, and inclusion of chemical inhibitors that increase the efficiency of the reprogramming process [2]. However, reprogramming largely remains dependent on the delivery and exogenous expression of one or more of the original Yamanaka factors.

\section{The newly emerging role of miRNAs in reprogramming}

In a recent issue of Cell Stem Cell, Morrisey and colleagues [3] report that iPSCs can be generated solely through the expression of a set of miRNAs, thereby avoiding all original Yamanaka factors for the first time. This breakthrough is destined to expand our understanding of the pathways that drive reprogramming. Using lentivirus-based expression of the miR-302/367 cluster to reprogram both mouse and human cells, Morrisey and colleagues [3] show that miRNA-based reprogramming proceeds faster than with standard fourfactor reprogramming. Consistent with this finding, pluripotency genes such as Sox2, Nanog and Rex1 are upregulated earlier in fibroblasts expressing the miR-302/367 cluster than in fibroblasts transduced with the four transcription factors. Using a mouse line expressing a reporter gene with the Oct4 promoter driving green fluorescent protein, the authors [3] also show that the endogenous Oct4 locus is reactivated to a greater extent following miRNA expression than without miRNA expression. This rapid induction of endogenous pluripotency genes in the majority of target cells results in a significantly more efficient reprogramming process, up to two orders of magnitude higher than standard fourfactor reprogramming. The authors [3] report that the miRNA-based approach can reprogram up to $10 \%$ of the input cells, although this could be an overestimation as only morphological criteria, and not pluripotency marker expression, were used to quantify the efficiency of reprogramming of human fibroblasts.

Several miRNA families are expressed exclusively and at high levels in embryonic stem cells (ESCs) [4]. ESCspecific miRNAs, such as the miR-290 and miR-302 
families, are directly regulated by the pluripotency factors Oct4, Sox2 and Nanog and are thus integrated into the core pluripotency network [5]. These miRNA families have important roles in ESC self-renewal and pluripotency, as knocking out either of the two key enzymes in miRNA biogenesis (Dicer and DGCR8) leads to defects in ESC proliferation and differentiation [6]. Inhibition of Dicer and DGCR8 also decreases reprogramming efficiency, indicating that miRNA biogenesis is essential to robust reprogramming [7].

The miR-302/367 cluster used by Morrisey and colleagues [3] is composed of five miRNAs, four of which (miR-302a-d) have the same seed sequence - a seven base pair stretch of nucleotides that determines target specificity. The miR-302 family belongs to a subset of miRNAs referred to as ESC-specific cell-cycle-regulating (ESCC) miRNAs that regulate the G1-S transition and can rescue the cell cycle defect of DGCR8-null ESCs [8]. Another set of ESC-specific miRNAs, the miR-290 family, has been shown to substitute for c-Myc expression during reprogramming [9]. Recent work has revealed that the miR-302 and miR-290 family members both target many genes in several pathways, such as cell cycle regulation (Cdk1na, Rbl2) and epithelial-mesenchymal transitions (RhoC and Tgfbr2) [9]. Many of these target genes are functionally important, as inhibiting them individually using siRNAs or chemical inhibitors can also enhance four-factor reprogramming [9]. The seed sequence of the other miRNAs used in the new report [3], miR-367, is different from that of the miR-302 family. Importantly, exclusion of miR-367 from the miRNA cocktail abrogated reprogramming. In fact, without miR-367, endogenous Oct4 is never activated, suggesting that this miRNA either directly or indirectly regulates Oct4 expression. Given the wide variety of cellular processes targeted by these miRNAs, it is likely that simultaneous suppression of multiple targets is key to their reprogramming ability (Figure 1).

\section{Potential mechanisms of miRNA-based reprogramming}

Although miRNA-based reprogramming did not require expression of any Yamanaka factors, reprogramming of mouse cells with the miRNAs did require the use of valproic acid (VPA), a histone deacetylase (HDAC) inhibitor. Interestingly, VPA and other HDAC inhibitors have previously been shown to enhance four-factor reprogramming [10]. Using fibroblasts derived from HDAC2 null mice, the authors [3] demonstrate that the effects of VPA are entirely dependent on the presence of this protein. Moreover, human fibroblasts express lower levels of HDAC2 than mouse fibroblasts, which may explain why miRNA-based reprogramming of human cells does not require VPA.

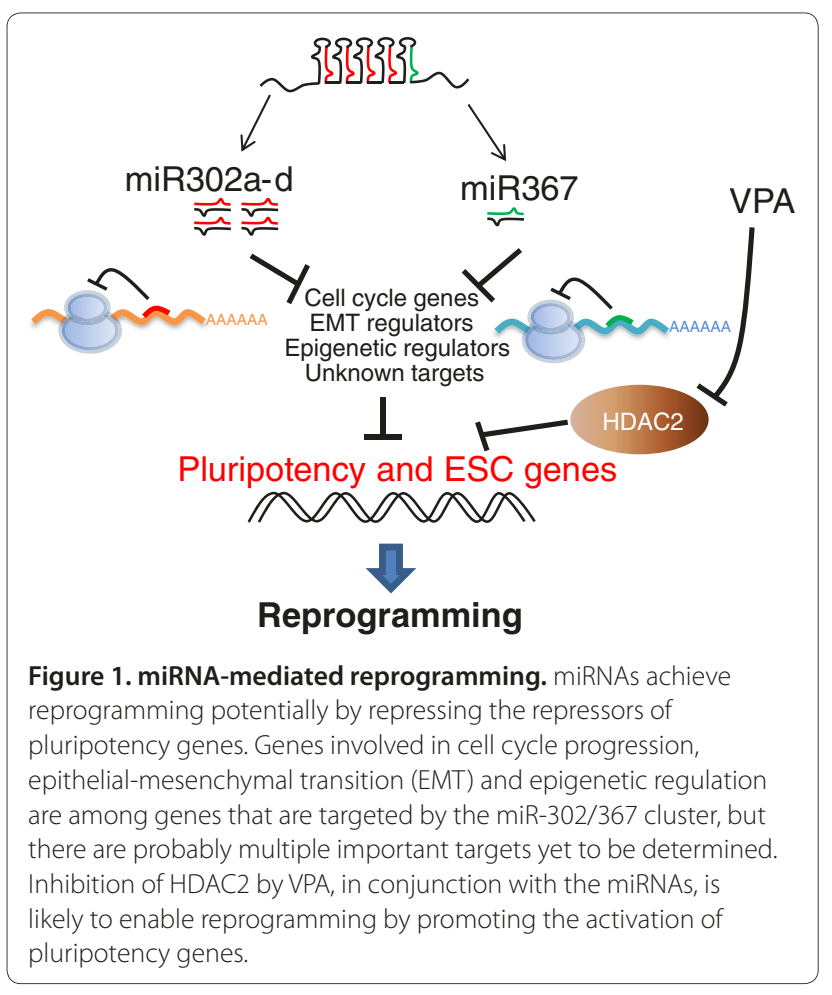

As in all standard retro- and lentiviral-based reprogramming methods, exogenous lentiviral miRNA expression is eventually silenced in the resulting iPSCs, when the endogenous pluripotency genes become reactivated. Because miRNAs primarily act as repressors of gene expression through mRNA degradation or inhibition of translation, it will be interesting to know how the endogenous pluripotency factors become activated during this process. In differentiated somatic cells these factors are kept silent by a combination of DNA and histone methylations; therefore, the miRNAs must somehow prompt the removal of these repressive chromatin marks [2]. A plausible scenario might be one in which the miRNAs target the enzymes that maintain these epigenetic marks. Inhibition of HDACs, which seem to be essential for miRNA-based reprogramming, at least in mice, may then shift the balance towards histone acetylation and transcriptional activation. Even then, how reprogramming is initiated in the absence of any strong transcriptional activator remains unresolved.

\section{The future of miRNA-based reprogramming}

Apart from the fascinating biological questions raised by this report [3], miRNA-based reprogramming has important practical implications. Alternative methods of miRNA delivery, such as transfections of miRNA mimics, are worth pursuing as a way to generate iPSCs with no genomic integrations. Although there are non-integrating methods of delivering the Yamanaka factors, the low 
efficiency of these approaches has hampered their wide adoption. As miRNA-based reprogramming seems robustly efficient, even non-integrating methods such as miRNA transfection may generate appreciable numbers of iPSC clones. If rapid and efficient reprogramming by transient miRNA delivery becomes a reality, routine iPS derivation for future clinical applications may rely entirely on this method.

\section{Abbreviations}

ESC, embryonic stem cell; HDAC, histone deacetylase; iPSC, induced pluripotent stem cell; miRNA, microRNA; VPA, valproic acid.

\section{Competing interests}

GQD is a member of the scientific advisory boards of iPierian and Verastem.

Published: 22 June 2011

\section{References}

1. Takahashi K, Yamanaka S: Induction of pluripotent stem cells from mouse embryonic and adult fibroblast cultures by defined factors. Cell 2006, 126:663-676.

2. Plath K, Lowry WE: Progress in understanding reprogramming to the induced pluripotent state. Nat Rev Genet 2011, 12:253-265.

3. Anokye-Danso F, Trivedi CM, Juhr D, Gupta M, Cui Z, Tian Y, Zhang Y, Yang W, Gruber PJ, Epstein JA, Morrisey EE: Highly efficient miRNA-mediated reprogramming of mouse and human somatic cells to pluripotency. Cell Stem Cell 2011, 8:376-388.
4. Houbaviy HB, Murray MF, Sharp PA: Embryonic stem cell-specific microRNAs. Dev Cell 2003, 5:351-358.

5. Marson A, Levine SS, Cole MF, Frampton GM, BrambrinkT, Johnstone S, Guenther MG, Johnston WK, Wernig M, Newman J, Calabrese JM, Dennis LM, Volkert TL, Gupta S, Love J, Hannett N, Sharp PA, Bartel DP, Jaenisch R, Young RA: Connecting microRNA genes to the core transcriptional regulatory circuitry of embryonic stem cells. Cell 2008, 134:521-533.

6. Wang Y, Baskerville S, Shenoy A, Babiarz JE, Baehner L, Blelloch R: DGCR8 is essential for microRNA biogenesis and silencing of embryonic stem cell self-renewal. Nat Genet 2007, 39:380-385.

7. Li Z, Yang CS, Nakashima K, Rana TM: Small RNA-mediated regulation of iPS cell generation. EMBO J 2011, 30:823-834.

8. Melton C, Judson RL, Blelloch R: Opposing microRNA families regulate self-renewal in mouse embryonic stem cells. Nature 2010, 463:621-626.

9. Subramanyam D, Lamouille S, Judson RL, Liu JY, Bucay N, Derynck R, Blelloch R: Multiple targets of miR-302 and miR-372 promote reprogramming of human fibroblasts to induced pluripotent stem cells. Nat Biotechnol 2011, 29:443-448.

10. Huangfu D, Maehr R, Guo W, Eijkelenboom A, Snitow M, Chen AE, Melton DA Induction of pluripotent stem cells by defined factors is greatly improved by small-molecule compounds. Nat Biotechnol 2008, 26:795-797.

doi:10.1186/gm256

Cite this article as: Onder TT, Daley GQ: microRNAs become macro players in somatic cell reprogramming. Genome Medicine 2011, 3:40. 